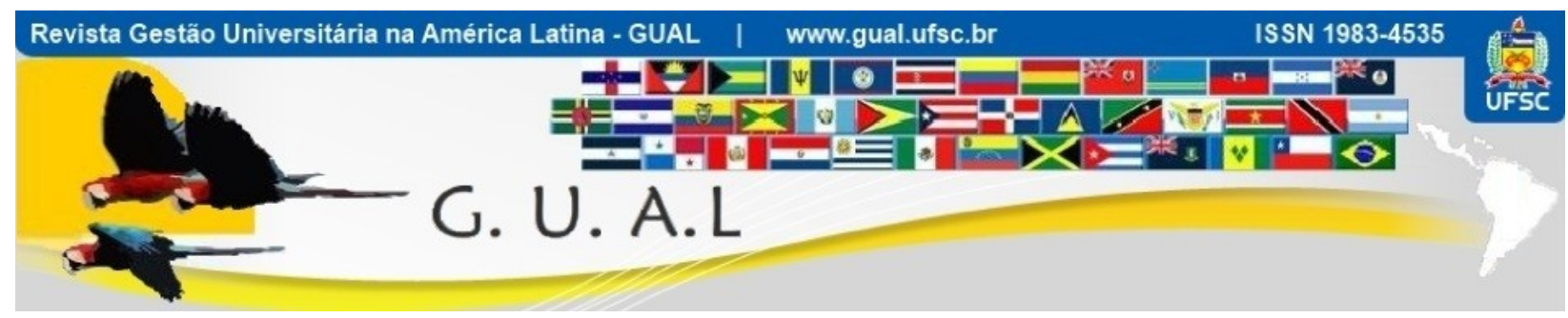

DOI: http://dx.doi.org/10.5007/1983-4535.2015v8n2p302

\title{
NUEVAS FORMAS DE REGIONALIZACIÓN DE LA EDUCACIÓN SUPERIOR EN AMÉRICA LATINA: LAS UNIVERSIDAD RED Y LOS CLUSTER UNIVERSITARIOS
}

\section{NEW FORMS OF REGIONALIZATION OF HIGHER EDUCATION IN LATIN AMERICA: THE UNIVERSITY NETWORK}

Claudio Antonio Rama Vitale, PhD

Universidad de la Empresa - UDE

claudiorama@gmail.com

Recebido em 15/junho/2014

Aprovado em 12/abril/2015

Sistema de Avaliação: Double Blind Review

Esta obra está sob uma Licença Creative Commons Atribuição-Uso. 


\title{
RESUMEN
}

En el presente artículo se analiza las distintas dinámicas de regionalización de la educación superior en América Latina como procesos históricos de espacios de poder al interior de las sociedades y de los sistemas universitarios y como ellas han reforzado y definido niveles de desigualdades en la construcción de capital humano. Se realiza una tipología y una contextualización de las formas de la regionalización universitaria pública y privada en América Latina y se formulan visiones sobre sus diferentes impactos en el empoderamiento y asociado a ello en la adquisición de competencias cognitivas. En el nuevo contexto regional y universitario se sostiene la irrupción de una diferenciación de esas formas, con el desarrollo de nuevas estructuraciones territoriales universitarias en red con mayor empoderamiento y búsqueda autónoma de articulación local, mostrando una de sus expresiones en los cluster universitarios.

Palabras claves: Universidad. Descentralización. Política educativa. Poder.

\begin{abstract}
In this article, the different dynamics of regionalization of higher in Latin America as historical processes of power spaces within societies and university education systems are analyzed, and focuses on how these inequalities have reinforced capital human regions. A typology of the forms of public and private university in Latin America regionalization is proposed, and their different impacts criteria formulated in flames skills acquisition, arising from the empowerment of educational processes. In the new regional and university context it is argued that institutional differentiation result of the emergence of a university network that promotes regionalization in greater empowerment, relevance occurs and coordination at the local level.
\end{abstract}

Keywords: University. Decentralization. Educational policy. Power. 


\section{EL CONTEXTO DE LA DESCENTRALIZACIÓN EDUCATIVA}

El análisis de la regionalización constituye uno de los ámbitos problemáticos conceptuales de los análisis sociales en América Latina en la cual se localizan visiones y enfoques contrapuestos, dada la polisemia del concepto y el carácter histórico en la conformación de las sociedades. En tal sentido es que se verifican interpretaciones divergentes de su dimensión y significación (Moncayo, 2002). En las últimas décadas al calor de las democratizaciones sociales, de los cambios en las tecnologías de la comunicación y de la expansión poblacional, han irrumpido nuevos abordajes del viejo debate que caracterizó al siglo XIX y parte del siglo XX en América Latina caracterizado por la confrontación ciudad campo, capital interior, Estado - regiones e inclusive civilización - barbarie. Los nuevos enfoques y valoración de las dinámicas regionales son múltiples y diversos, destacándose aquellos asociados al desarrollo y el empoderamiento (Putnam, 1993, Urteaga, 1993), a su rol como mecanismo de apaciguar los conflictos y presiones sociales a través de transferir conflictos a los ámbitos descentralizados (Huntington, 1975) o a las dinámicas económicas actuales asociadas a la construcción de capacidades, ventajas competitivas locales y redes de aprendizaje (Lundvall, 2002). Ellas expresan perspectivas teóricas que impulsan nuevas configuraciones de las relaciones entre el Estado y la sociedad. Ellas sin embargo remiten al enfoque hegeliano de relación entre la sociedad civil y la sociedad política y como se construyen las sociedades, por lo que la regionalización también impacta y determina las formas a partir de las cuales se construyen los sistemas educativos.

La regionalización tiene su basamento en el federalismo como raíz filosófica de enfoque liberal que la presenta como contrapeso de poder en la construcción de las sociedades y que ha sido expresada en el espíritu de la Constitución de los Estados Unidos. Inversamente los enfoques centralistas remiten a concepciones que otorgan un rol central al Estado en la construcción de la Nación y de su identidad nacional. Ellos tienen su raigambre en la tradición francesa de la construcción política de la sociedad. Esta segunda vertiente ha sido dominante en América Latina, dado el carácter colonial centralista de la dominación como raíz de la construcción de las naciones. En su transferencia a la dinámica universitaria ello se expresó en el modelo urbano, asociado al Estado, orientado a la construcción de la nación a través de una universidad profesionalizante y sociocultural y que para ello utilizó el modelo de universidad francés o napoleónico. Así, mientras que en Estados Unidos no se crearon universidades nacionales, siendo la regulación de los Estados y desde su génesis hubo universidades 
privadas, en la región, siguiendo la tradición napoleónica, las universidades han sido creadas como instituciones con un sesgo como instituciones nacionales y dependientes del Estado nación.

La universidad latinoamericana fuera del momento de la independencia tendió a ser una universidad nacional o universidad mayor, con un paradigma monopólico en su conformación como servicio de elite y su carácter de institución importada de origen colonial. Hubo un impulso regional en el siglo XIX con la construcción de algunas burguesías regionales con relativa capacidad de autonomía, muchas veces asociado a su distancia de las capitales y a sus poderes de exportación regionales y su inserción internacional. En el siglo XX con la expansión y democratización de la educación superior y la construcción de poderes estatales en las regiones, ha aumentado la diferenciación institucional y la construcción de sistemas universitarios con mayor nivel de diversidad institucional, incluyendo pluralidad de accesos, de niveles y de modalidades. Su dinámica histórica ha estado marcada por fuerzas centrífugas o descentralizadoras y fuerzas centrípetas o centralizadoras, como ejes de la conformación de los sistemas de educación superior. Los resultados de esas fuerzas han impactado en las formas y dimensiones de la regionalización (Martínez, 2002). Ello ha sido resultado de la existencia de espacios en pugna de intereses y dinámicas que han ido construyendo a su vez diversidad de formas de regionalización, de empoderamiento, de especializaciones y de universidades e instituciones superiores en términos de su expresión nacional, federal, provincial, departamental, distrital, regional o municipal con sus diversas y respectivas localizaciones en el nivel primario, secundario y terciario de la organización de los Estados. Al tiempo estos también se han expresado diferenciadamente con múltiples y diversos niveles de coordinación, subordinación o autonomía y diferenciación asociados a los distintos niveles de centralización, desconcentración, descentralización o de formas híbridas o mixtas de organización estatal.

Los procesos de regionalización en América Latina no han sido espacios de consensos, como no lo han sido tampoco el federalismo y el centralismo, sino un resultado de pugnas y de política. Los escasos recursos de las regiones y la centralización de los ingresos nacionales de raíz tributaria aduanal en su génesis, también han impactado las formas de regionalización y limitado los empoderamientos que la regionalización implica. Contemporáneamente tanto los impulsos como las resistencias han agregado nuevos enfoques. Para algunos la regionalización en lo eductivo constituye un «paradigmas tecnicista y eficientista - propio de 
la Nueva Gestión Pública - que se impuso desde los 90 y que propende a poner a las universidades al servicio de la producción y de los proyectos políticos provinciales....(donde) las universidades provinciales se han hecho eco de muchas de las consignas de las políticas neoliberales instauradas a partir de los 90, (mostrando) una gran dependencia del poder político provincial, lo que pone en serio riesgo la autonomía» (Menghini, 2007). Inversamente, otros enfoques educativos de la regionalización han valorado los procesos de eficiencia en la gestión (Barry, 2005), más allá de destacar también su impacto en el empoderamiento y el mejoramiento de la pertinencia y la calidad. Estos debates del pasado han regresado con nuevos enfoques. Para Martínez (2002) la tensión alrededor de la regionalización de la educación superior, más allá de los distintos modelos históricos de sus sociedades, se retoma cuando una sociedad crece y se hace más compleja.

La construcción y el desarrollo de las universidades regionales, provinciales o de sedes en el segundo y tercer nivel de los Estados en América Latina ha constituido un proceso altamente diverso a nivel de los distintos países y regiones, y que en el contexto de la masificación y diferenciación institucional, muestra las tensiones alrededor del empoderamiento, de la pertinencia y de la autonomía regional. La tensión de la regionalización es resultado de ser el espacio de la conformación de los sistemas nacionales de educación superior pero con formas distintas. En tal sentido, la construcción de estos sistemas está asociada a conflictos y tensiones alrededor de las formas de la descentralización, desconcentración o de los modelos mixtos o híbridos (Martínez, 2002). Ello tanto en el sector público como en el privado, si bien en el caso de la regionalización de la universidad privada se estructura bajo lógicas donde el mercado es el agente asignador dominante, y donde también ha estado sujeta, aunque en menor proporción a decisiones políticas de regulación que han habilitado, protegido o negado sus niveles de regionalización y de empoderamiento. Las formas de concentración universitaria en grandes grupos han limitado las formas de la regionalización privada con mayor nivel de empoderamiento en la gestión y han estructurado procesos de regionalización con alta concentración institucional como en Brasil o México.

En el caso de la regionalización del sector universitario público, la construcción de universidades en el segundo nivel del Estado, ha sido un proceso con dimensiones más políticas y basadas en concepciones ideológicas del servicio público y en las dinámicas de luchas de poder al interior de estas instituciones. 


\section{LA TRADICIONAL INEQUIDAD REGIONAL UNIVERSITARIA}

La inequidad regional en América Latina ha sido una de los componentes que ha caracterizado a la educación superior desde sus inicios. Tal dinámica ha sido menos analizada en la región (Martinez, 2002) e inclusive ha sido soslayada en algunos buenos estudios sobre la propia desigualdad que dan más atención a los enfoques sociales (género, raza o condición social) que a los enfoques regionales en el análisis de la inequidad (Solana, 2005; Ezcurra, 2011). Los estudios de las desigualdades universitarias en tal sentido, se han centrado mayoritariamente en los circuitos desiguales de calidad, de género, de cultura o raza y de la condición socioeconómica de los estudiantes. La problemática de la regionalización es vista desde su dimensión política e histórica, desde un paradigma por el cual el Estado nación se organizó sobre un enfoque de la centralización educativa. En un estudio se sostiene que "los problemas "regionales" y el "centralismo" tienen que ver tanto con la falta de reconocimiento de las regiones y provincias como "sujetos políticos" (regionalización) como con la escasa transferencia y distribución de poder político administrativo y fiscal (descentralización) a los organismos subnacionales (llámese, regiones, provincias y municipios)". Para el caso de éste país, se sostiene que la regionalización nunca fue pensada para transformar a las regiones y provincias en sujetos políticos (por ejemplo mediante elección de sus propias autoridades), por lo que las medidas de descentralización (o las propuestas para su fortalecimiento) actúan sin reconocer las dinámicas políticas y que por ende están siempre limitadas. En tal sentido se plantea que los voluntarismos por descentralizar, al no enfrentar los problemas estructurales de poder que padecen las regiones tales como la fragmentación intrarregional, disputas entre provincias, historicidad de dependencia de regiones, niveles de gestión, etc., no solucionan sus problemas de fondo (Montecinos, 2013).

La regionalización se ha centrado en los últimos años, en su rol asociado a la creación de capacidades articulado a la creciente competitividad de los ámbitos regionales a la escala mundial y correlativamente a las demandas políticas de gestión y empoderamiento de los recursos humanos. Bajo este enfoque, la regionalización educativa se asocia al acceso y egreso educativo a nivel local y a la construcción de pertinencias como un indicador de la calidad de los aprendizajes para los diversos sectores sociales y productivos. Bajo este enfoque de creación de capacidades la regionalización es más eficiente dada la cercanía con el objeto de trabajo en el entorno regional y la mejora de las competencias apropiadas. 
La desigualdad regional, en tanto una de las expresiones de las desigualdades educativas, refiere a las diferencias en la educación ofrecida y demandada entre las diversas áreas geográficas, entre el medio rural y el medio urbano, entre las grandes ciudades, las capitales departamentales y las ciudades menores en términos de calidad, cantidad, diversidad y pertinencia. Refiere a diferencias que impactan en el desarrollo económico de las regiones al dotarlas de menor capital humano y con menores habilidades cognitivas y por ende inferiores niveles de productividad y con ello de oportunidades de trabajo y de inversión.

La problemática de la regionalización adquiere nuevas particularidades en el actual contexto donde se prioriza que la calidad educativa es una variable más importante que la cantidad de estudiantes o egresados en los procesos de formación (Hanushek, 2011; Heredia, 2011). Bajo este enfoque las desigualdades regionales se asocian a las desigualdades de calidad por los menores niveles de competencias adquiridas y por ende menores niveles de capacidades laborales. La baja regionalización en cantidad de recursos humanos y la desigual regionalización en calidad es una particularidad de América Latina y el Caribe derivado, en parte, de que el sistema educativo ha sido la expresión de un modelo económico y político con alto sesgo macro cefálico que además ha sido reforzado por las estructuras de poder particulares de las universidades. Ello derivó en una dinámica educativa caracterizada por una desigualdad en el acceso, la cobertura y el egreso a escala regional, así como también de una escasa pertinencia y calidad de los programas localizados en la mayoría de las sedes del interior respecto a los programas ofrecidos a nivel de las capitales por las mismas universidades. Ello respondió a muy diversos motivos, éntrelos cuales la baja autonomía, recursos, escalas y pertinencia en la gestión de las sedes y subsedes regionales, así como de los mecanismos de distribución del poder y de las concepciones educativas dominantes.

Tales situaciones han reforzado dinámicas socioeconómicas excluyentes espacialmente, en tanto la concentración de la matrícula de las universidades públicas y privadas en las capitales de los países determinó accesos reducidos y de elite en las capitales de los estudiantes de los estratos de ingresos más altos provenientes del interior de los países. Esta inequidad educativa retroalimentó la inequidad socioeconómica en tanto sólo los sectores de altos ingresos del interior pudieron asumir los costos de traslado de sus estudiantes. Además en tanto muchos de ellos no regresaron a sus lugares de origen, hubo un drenaje de recursos humanos de las regiones del interior hacia las capitales, lo cual alimentó que la educación facilitara la reproducción de las estructuras desiguales de ingreso. 
La universidad latinoamericana como matriz cultural e institucional nació como una institución de elite, perviviendo hasta fines del siglo XX como una universidad urbana, de hombres blancos y de estudiantes de altos recursos. Más allá de las reformas de la independencia en el siglo XIX, de algunos esbozos regionales a fines de ese siglo y principios del XX, de los movimientos autonomistas de la Reforma de Córdoba, recién desde fines de los 60, se ha democratizado con el inicio de la masificación de su cobertura (Rama, 2009) y de su diferenciación institucional. En este contexto la masificación y diferenciación contribuyó a reducir algunas de las desigualdades tradicionales, entre las cuales también las de carácter espacial que son el centro de nuestro análisis. Esta relativa regionalización permitió que la cobertura universitaria aumentara en la dimensión acontecida y su evolución posterior fue una de las formas de la diferenciación institucional junto a la educación privada, la educación a distancia y la educación tecnológica terciaria.

La regionalización no se inició meramente como resultado de la expansión del sistema universitario, sino que ella respondió a demandas de democratización política, de acceso y a concepciones asociadas a la descentralización así como a una expansión de las economías regionales que comenzaron a reclamar recursos humanos más capacitados, y que se expresó en demandas de empoderamiento de las regiones en casi toda América Latina. Esta regionalización también fue favorecida por una dinámica competitiva entre las instituciones educativas sobre todo privadas buscando aumentar sus coberturas y sus escalas y regionalizandose buscando cubrir demandas de acceso insatisfechas. También ha habido nuevas políticas estatales con un cambio en los enfoques tradicionales de desconcentración de la educación superior hacia una mayor atención a formas de descentralización.

La tradicional tensión pública privada de la reforma de la mercantilización y del modelo binario (Rama, 2006), ha estado en el centro de las nuevas dinámicas de regionalización que facilitaron nuevas tipologías institucionales y la conformación de más amplios subsistemas de educación superior con instituciones con mayores escalas nacionales.

El aumento de las demandas de acceso en las regiones ha sido un proceso autónomo que se ha enfrentado a las diversas desigualdades e inequidades en el funcionamiento de los sistemas universitarios que han limitado la masificación de la cobertura y han mantenido las desigualdades existentes (Castrejón, 1992). Por ello la regionalización no ha sido un proceso lineal, sino un camino de múltiples resistencias al interior de las estructuras educativas e inclusive de los diversos actores académicos o políticos urbanos reticentes a perder poder, 
recursos o calidad ante esos procesos de enseñanza. En este sentido, el curso y las características que han asumido la regionalización desde su inicios han sido determinadas por las variables políticas al interior de los países, por los sistemas universitarios y sus marcos normativos, por las formas de ejercicio del poder universitario, por las orientaciones de las universidades dominantes, así como por las ideas y paradigmas existentes sobre la democratización de los accesos en el interior.

Más aún, las modalidades y dimensiones de la regionalización universitaria en América Latina han sido determinadas por las concesiones de los actores dominantes, por las luchas locales frente a los poderes centrales, por las dinámicas internas de las estructuras monopólicas y autónomas universitarias en varios países de la región, así como por las formas de la competencia interuniversitaria a escala de los países. Han sido estas dinámicas las que a su vez han marcado las resistencias y por ende las formas que ha asumido la diferenciación institucional como parte de la regionalización universitaria.

\section{LA DIFERENCIACIÓN DE LOS MODELOS DE REGIONALIZACIÓN UNIVERSITARIA}

En general en América Latina, las demandas de acceso han impulsado la diversificación institucional pública y privada, gracias a la cual se han reducido las desigualdades universitarias entre el interior y las capitales, así como entre los docentes y los mercados laborales. Sin embargo, las formas de esa regionalización en los diversos países han sido diferentes en términos de ingreso, permanencia y egreso, y por ende con un impacto distinto en la construcción de las capacidades locales, en los niveles de empoderamiento local y en los niveles de calidad de la enseñanza en las regiones.

Los enfoques universitarios tradicionalmente han diferenciado la regionalización bajo dos orientaciones para expandir la cobertura:

A: Desconcentración universitaria. Esta forma no transfiere poder educativo y tiene menores niveles de pertinencia y calidad. Tiende a producir sistemas más jerarquizados y estructurados a través de sedes al interior de las universidades o de centros de apoyo asociados a ofertas de educación a distancia. Bajo esta modalidad la lucha por el empoderamiento y recursos, y por ende la creación de capacidades se produce al interior de las estructuras institucionales de las universidades y sobre estándares estructurados desde la sede central. 


\section{NUEVAS FORMAS DE REGIONALIZACIÓN DE LA EDUCACIÓN SUPERIOR EN AMÉRICA LATINA: \\ LAS UNIVERSIDAD RED Y LOS CLUSTER UNIVERSITARIOS \\ DOI: http://dx.doi.org/10.5007/1983-4535.2015v8n2p302}

B: Descentralización universitaria. Esta forma tiende a promover empoderamientos socio-académicos y por ende mayores niveles de pertinencia. Ella tiende a fragmentar el sistema en múltiples actores e instituciones y se expresa en la creación de instituciones en las regiones. En este caso las dinámicas de empoderamiento y búsqueda de más capacidades se asocian a mayores niveles de libertad con sus estándares propios y una mayor orientación hacia la creación de alianzas locales y regionales, o inclusive internacionales.

A partir de dos formas de regionalización se constata que se está produciendo un cambio con una mayor entonación hacia las formas de descentralización universitaria, con nuevas expresiones que superan el modelo dual referido. Ello está asociado a nuevo curso de política y tipologías, a las tecnologías de comunicación, una mayor dinámica competitiva que facilita acuerdos entre las instituciones de educación superior, así como el desarrollo económico, social e institucional de las fuerzas regionales en los diversos países. Todo ello no sólo impulsa mayores niveles de empoderamientos regionales, sino nuevas formas de regionalización universitaria y cuyo trasfondo es un cambio en la relación entre el Estado y regiones en lo "universitario".

El cambio político precede, ya que finalmente la regionalización de la educación superior es un proceso político de negociación y conflicto con distintas formas institucionales de organización, resultado de como se procesan las tensiones alrededor de la construcción de capital humano y de las luchas políticas alrededor del grado de empoderamiento y autonomía de las regiones y donde la universidad es una variable con distinto grado de adscripción en esa tensión. La forma en cómo se resuelven esas tensiones en cada país, es la variable determinante de la regionalización de la educación superior.

En las últimas décadas la dinámica de la regionalización ha cambiado permitiendo una expansión de la cobertura pública como también privada con la irrupción de nuevos particularismos y diferencias frente a las modalidades tradicionales de la regionalización, complejizando el tradicional modelo dual. A continuación analizaremos algunas de las distintas formas de regionalización de la educación superior, atendiendo a sus particularidades institucionales, verificando un aumento de la diferenciación de las tipologías tradicionales de regionalización con mayor intensidad de las formas de descentralización y un cambio en las formas de desconcentración.

Hay una discusión conceptual sobre la eficiencia de la descentralización frente a la eficiencia de modelos centralizados en el ámbito universitario. La forma centralizada concibe 
la calidad de los aprendizajes por la interdisciplinariedad y la articulación de los servicios al interior de los campus universitarios. Este enfoque de la centralización concibe una regionalización similar a la del sector de los servicios de salud donde se propende a un sistema integrado en el cual se derivan los casos y donde los centros de atención comunitarios, los centros intermedios, los hospitales generales y los centros de alta especialización funcionan como un proceso sistémico y articulado de servicios. Esta visión concibe un sistema jerarquizado en educación como en salud, aún cuando para algunos ello inclusive no deriva necesariamente en una concentración de los servicios en las grandes ciudades sino a un amplio entramado de movilidad, redes y especialización. En el caso de los sistemas de salud, la complejidad de los sistemas de traslado en términos sanitarios y las eficiencias de las altas escalas por los costos de los centros de salud, impulsan su centralización asociado a patologías de alta especialización y que tienen poca incidencia poblacional. Por su parte el enfoque de la descentralización universitaria refiere a la cobertura, al acercamiento a los estudiantes y a la importancia de la pertinencia en la calidad del aprendizaje.

\section{LOS ENFOQUES DE RED EN EL ANÁLISIS DE LA REGIONALIZACIÓN}

Los nuevos enfoques de la educación superior en red superan la dicotomía referida desconcentración - descentralización - como ejes de la regionalización. Bajo los nuevos paradigmas la eficiencia universitaria se plantea en cómo lograr una regionalización con mayores niveles de calidad. La irrupción de la "universidad red" muestra como las tradicionales formas centralizadas asi como descentralizadas y desconcentradas a la vez que se van moviendo desde enfoques centrados en la enseñanza a enfoques centrados en el aprendizaje tienden a favorecer dinámicas en red. Concibe una asociación entre empoderamiento, cooperación en red y especialización universitaria regional. Concibe la necesidad de lógicas sistémicas de articulación regional y nacional entre los diversos subcomponentes que faciliten una mayor regionalización de los accesos y egresos y en igualdad de condiciones.

La creciente creciente irrupción de dinámicas sociales en red (Castells, 2001, 2012) impulsa procesos de aprendizaje al interior de esas redes en tanto la educación es un fenómeno que se produce a través de las diversas interacciones entre los actores al interior de organizaciones y estructuras que se conforman como comunidades de aprendizaje. Las redes 
son los ámbitos de apropiación de información que se desarrollan a través de interacciones para viabilizar el aprendizaje, con sus particularidades dadas por las propias redes y los tipos de tecnologías que median a las personas y sobre las cuales se estructuran los procesos del diálogo educativo (Freire, 1987).

Definimos tres tipos de organizaciones de redes sociales con sus diversas lógicas territoriales, sus diferenciados niveles de distribuir el poder, de intercambio y creación de conocimiento y gestión de los recursos, y con distintos niveles de eficacia, de distribución de poder y de creación de conocimiento. Identificamos un primer modelo centralizado de tipo jerárquico y cuya expresión regional universitaria se asocia a sedes. Un segundo modelo llamado descentralizado remite a una dinámica de universidades con doble jerarquización de sedes como nodos con sus propias subsedes. Y un tercer esquema, en red sin jerarquización sin centros de poder. Analizaremos a continuación una categorización más amplia de estas formas de regionalización universitaria para el caso de América Latina entre modelos universitarios centralizados y fragmentados (desconcentrados), descentralizados y en red.

\subsection{LOS MODELOS CENTRALIZADOS}

El arquetipo de universidad nacional localizada en las grandes ciudades y con un fuerte dependencia del Estado en el siglo XIX y que tenía como centro la construcción de las sociedades, promovió la "Universidad de los doctores" centrada en la formación de profesionales para el Estado y centrada en la creación de la identidad y la cultura nacional, que promovió un modelo centralizado que limitó la diferenciación regional. Fue herencia de la universidad colonial expresión del poder colonial donde la corona era la figura máxima de estas Universidades Reales o la Iglesia en las Reales Pontificias y sus currículos y enfoques seguían los paradigmas de Salamanca. Pudieran ser clasificadas como instituciones independientes pero adscritas en red a las universidades en España. A diferencia la corona portuguesa que no constituyó una red institucional sino una sola institución, la Universidad de Coimbra como centro de formación, la colonización española creó un modelo centralizado con nodos independientes en los virreinatos y capitanías, inicialmente sujetos al control curricular de Salamanca.

En los inicios del siglo XIX, Simón Bolívar estatizó las universidades religiosas e impulsó la creación de universidades en los diversos departamentos de la Gran Colombia, que marcaron la impronta de los procesos de regionalización universitaria en Venezuela, 
Colombia, Perú (Arequipa y Trujillo) y Ecuador donde se crearon universidades regionales. En este camino, a fines del siglo XIX y principios del XX la expansión de las burguesías regionales impulsó nuevas fundaciones de universidades regionales como Antioquia, Zulia, La Plata, Guayaquil o Cuenca. En el siglo XX, la autonomía y cogobierno, conformadas como derivación de la Reforma de Córdoba, se constituyó en un modelo institucional urbano que tendió a limitar la diferenciación institucional y la descentralización universitaria, y facilitó la expansión de la matricula y desconcentración regional de las universidades a través de la creación de sedes promoviendo un mayor nivel de acceso. En algunos casos donde había universidades regionales creadas previamente, se propendió a un lógica universitaria sistémica donde la universidad de la capital, como "Nacional" o "Mayor", se colocaba por encima de los intereses regionales (Burbano, 2008).

El paradigma educativo que soporta la centralización de los servicios y ofertas universitarias refiere al concepto de universidad compleja que integra y unifica todos los conocimientos y campos disciplinarios y que centraliza espacialmente las ofertas y cuerpos académicos con miras a lograr eficiencias de escalas económicas y articulaciones académicas que posibiliten mayores escalas de los sistemas de apoyo académico y la creación de nuevos campos de conocimiento de tipo interdisciplinario, multidisciplinario o transdisciplinario resultado de la cercanía. Tal enfoque está en el sustento de los modelos tanto altamente centralizados y macro cefálicos como en el los campus universitarios. Estas redes centralizadas se caracterizan por poseer un punto en el centro en el cual se concentra el flujo de información de toda la red y cuyos distintos nodos están integrados y jerarquizados espacialmente.

Los campus universitarios de inspiración americana constituyen un ejemplo de estos modelos. Sin embargo, cabe diferenciar los campus universitarios de las macro universidades latinoamericanas en tanto cumplen roles y tienen características altamente diferenciadas, más allá de que muchas veces pueden ser los mismos. En los campus se tienden a asumir una escala máxima de tamaño eficiente, están fuera de las grandes ciudades, son de estudiantes de tiempo completo y están concentrados en la investigación. Las universidades latinoamericanas en su mayoría desarrollaron campus desde los años 40 y 50 .

Desde el punto de vista de las eficiencias de escala se concibe que la centralización de los diversos servicios de apoyo como bibliotecas, auditorios, ámbitos deportivos, comedores y servicios estudiantiles, laboratorios, etc., permiten que esas inversiones sean más eficientes y 
beneficien a mayores públicos universitarios. Ellos a su vez permiten surgir múltiples sinergias académicas. Los campus sin embargo, no necesariamente se asocian a universidades de gran tamaño de matricula sino a una especial conformación de la dinámica académica, con estudiantes de tiempo completo y un perfil centrado en la investigación. Ellos además permiten la conformación de una periferia institucional universitaria como oficinas centrales de evaluación, de propiedad intelectual, de gestión curricular, que se constituyen en uno de los cinco ejes de la universidad innovadora (Clark, 2000). Es un modelo que se ha expresado en campus universitarios, fuera de las grandes ciudades y en espacios amplios de terrenos. Ellos han permitido alta movilidad estudiantil y docente entre unidades académicas con estructuras de créditos académicos, flexibilidad de recorridos e facilitan un funcionamiento de la base académica en Departamentos. Para impulsar estas dinámicas es que los campus se localizan fuera de las ciudades grandes, privilegiando con ello una alta dedicación de los estudiantes de tiempo completo.

En los casos de América Latina, bajo la gratuidad y el cogobierno con financiamiento público, la centralización de las universidades, se expresó en macro universidades instaladas en las grandes ciudades y dominantemente en la malla urbana, integrando dominantemente estudiantes de grado y de horarios nocturnos, y a la vez en la creación de sedes en el interior de los países o regiones. Estas dinámicas de localización, gratuidad, acceso escasamente selectivo en muchos países, estudios nocturnos y regímenes altamente flexibles, han contribuido a reforzar los modelos centralistas. La selectividad fue inversamente un mecanismo de reducción de la cantidad de sedes.

\subsection{LOS MODELOS UNIVERSITARIOS FRAGMENTADOS}

La organización territorial universitaria de tipo fragmentada, constituye la modalidad dominante en América Latina de regionalización de los sistemas universitarios. Ellos se soportan en dinámicas jerarquizadas con una dinámica permanente de conflictos de poder alrededor de los recursos, el empoderamiento y la autonomía entre los nodos centrales y las terminales locales, pero que requieren sedes para aumentar poder, recursos y cobertura.

\subsubsection{La desconcentración en subsedes}

El modelo universitario centralizado tiende a regionalizarse conformando una universidad sistema en la cual se diferencia y regionaliza a través de unidades 
desconcentradas como sedes o subsedes fuera de su sede central o de su ciudad sede. La universidad es la cabeza de un sistema regional de educación superior constituido a su interior mediante sedes y Facultades. Fue la forma dominante por la cual los Estados impulsaron la regionalización y el acceso a la educación superior en las regiones, mediante la desconcentración regional de algunas de las ofertas al interior de las instituciones. Esta desconcentración ha tenido múltiples denominaciones y dimensiones como filiales, subsedes, sedes regionales, seccionales, etc., con pluralidad de denominaciones y de niveles de delegación de poder y recursos en la gestión administrativa y académica. En general la desconcentración ha estado asociada a las escalas y dimensiones de los nodos centrales. Cuando más grande es la universidad, y por ende mayor poder, la regionalización se ha realizado vía desconcentración. Al tiempo ambos componentes - escala de las instituciones y sedes - se han retroalimentado en una dinámica interna que ha concentrado poder y recursos gracias a la desconcentración. Este tipo de dinámica fue funcional a un patrón de ejercicio del Poder Estatal centralizado con pocas universidades en general de elite y que fue uno de los mecanismos de democratización. La dinámica centralista universitaria favorecida por la autonomía y el cogobierno a la vez impulsó la desconcentración como mecanismos de regionalización y democratización, pero a la vez de un empoderamiento regional limitado en las sedes. Este fue el eje dominante de la regionalización de la universidad pública en América Latina en el siglo XX, y estuvo asociado a la autonomía y el cogobierno con baja diferenciación institucional. Bajo este paradigma la Universidad impulsó su desarrollo constituyéndose en instrumento de políticas centralizadoras en el ejercicio de su poder sobre la nación, a través de un escaso empoderamiento de los accesos regionales.

Este modelo de desconcentración, impulsó una localización espacial altamente distribuida, diferenciando también la calidad de las ofertas universitarias entre el nivel local y el centro. En este modelo, la dinámica educativa es una dinámica de poder y de tensión entre la unidad desconcentrada y la unidad centralizada, donde lo educativo es una derivación de esas dinámicas políticas y donde el marco normativo, la diferencia de tamaño y de estructuras de gobierno, siempre imponen una relación de dependencia. El empoderamiento es desigual por los bajos recursos y la poca capacidad de iniciativa en las terminales, derivado de la ausencia de competencia, libertad y recursos. El modelo de organización desconcentrado ha favorecido una división del trabajo entre las sedes y las filiales, con una permanente discusión de poder y de recursos, con niveles de calidad asociados a esos debates y tensiones. 
Este modelo jerárquico lineal, en algunos casos, dadas sus tensiones internas, ha derivado en su transformación hacia modelos descentralizados mediante la ruptura institucional y la autonomización de las sedes. Tal situación aconteció en Panamá donde la regionalización de la Universidad de Panamá a través de la desconcentración derivó en una ruptura y una lógica de descentralización. La Universidad de Panamá siguiendo dinámicas institucionales endógenas, de demandas de las regiones en función de su tamaño y de los recursos económicos, así como de sus propios cuerpos académicos, llevo adelante la creación de sedes fuera de la capital, con múltiples limitaciones de recursos, tensiones de empoderamiento y diferencia de culturas educativas organizacionales. Las presiones políticas en el ámbito parlamentario, llevaron a la autonomización de la sede de Chiriquí al norte del país y su constitución como Universidad de Chiriquí en el año $1995 .^{1}$ Este pasaje de sede regional como ámbito desconcentrado a una institución autónoma como unidad descentralizada aumentó la diversidad y promovió la lenta conformación de un sistema universitario público. La conformación del sistema de educación superior y su diferenciación no sólo tuvo su impulso desde la diferenciación regional, sino también posteriormente por una diferenciación disciplinaria, con la autonomización de su Facultad de Ingeniería y su transformación en Universidad Tecnológica de Panamá (UTP) en 1981. La nueva universidad a la vez promovió posteriormente una regionalización a través de modelos de desconcentración al interior de la UTP, al crear siete sedes regionales.

La desconcentración en la inmensa mayoría de los casos ha sido con inferiores niveles de calidad, lo cual también ha incentivado las rupturas institucionales como mecanismo para igualar las condiciones de acceso y egreso y sobre todo de poder y cobertura. En Bolivia, de las 11 universidades públicas, 9 son Departamentales centradas en sus regiones y entre todas sumando 108 sedes y subsedes, en el marco de ausencia de un sistema nacional de aseguramiento de la calidad (CEUB, 2014). En muchas de estas subsedes, como en otros países, los requerimientos de ingresos son menores que en las sede. A diferencia, otras instituciones han propendido a que la desconcentración en subsedes sea igualitaria en términos de calidad, lo cual ha permitido crear dinámicas sistémicas de mejoramiento de la calidad en forma colaborativa y articulado. El Instituto Tecnológico de Monterrey constituye

\footnotetext{
${ }^{1}$ El nacimiento de la Universidad Autónoma de Chiriquí, culmina un proceso de más de 45 años. Se inicia con los cursos de verano en la Ciudad de David en 1951 por la Universidad de Panamá, que en 1958 se convertiría en Extensión Universitaria, en 1969, en Centro Regional Universitario de Chiriquí (CRUCHI) y finalmente en 1995, por Ley en Universidad Autónoma de Chiriquí, (UNACHI).
} 
un caso referencial de conformación de una amplia red con más de 26 instituciones a nivel de todo México con iguales niveles de calidad y de empoderamiento. Luego de una expansión caótica con distintos niveles de calidad y estándares en los campus, se estableció un modelo de estándares iguales, autonomía de gestión y sistemas de información integrados con un Board de Consejeros independientes y de la región en cada uno de ellos, con centralización del diseño de los planes de estudio, los métodos administrativos, la misión y los controles de calidad para ofrecer programas similares (Rangel, 2014). En tal contexto se visualizó como aspecto negativo, la creación de burocracia promovida por la centralización, más allá de la incorporación de tecnologías de gestión para subsanar el tamaño de las sedes pequeñas. Pero también, la ausencia de escalas en algunas sedes y pon ende la escasa capacidad de cumplir el estándar de calidad sistémico, llevo a su cierre y a su oferta mediante una diferenciación institucional a través del TEC Milenium y no del TEC. Se concibió y creó otra universidad con un menor nivel de estándares para no deteriorar la reputabilidad de la institución madre.

Una alta desconcentración con múltiples sedes, a medida que crece en filiales impacta en varias dimensiones en la totalidad de la estructura en términos de recursos, poder y niveles de efíciencia de los servicios. Se podría concebir un ciclo marcado por caída de los niveles de eficiencia sistémicos y conflictos permanente de distribución de los recursos asociado al mantenimiento del modelo bipolar centro - sedes, ruptura de las estructura organizacionales desconcentradas con la transformación de algunas de éstas en unidades separadas descentralizadas y pasaje desde modelos centralizados y desconcentrados a modelos de funcionamiento en red con un aumento de la incorporación de tecnologías de comunicación en la gestión y la prestación de los servicios mediante el impulso a la creación de otras universidades que se posicionan en mayores niveles de calidad o pertinencia diferenciados que no cubre la institución madre primigenia.

\subsubsection{Dualidades de niveles de regionalización en la educación superior}

Históricamente en algunas zonas de América Latina, derivado del marco de política establecida por Simón Bolívar en 1826-27 en la época post independencia, el modelo universitario se expresó en forma fragmentada a escala de las naciones entre las capitales y las regiones. Ella implico el establecimiento de servicios descentralizados por Departamentos pero a la vez subordinados en diversas formas. En algunos casos implico la creación de universidades en los diversos departamentos pero en un marco institucional jerarquizado por 
el cual la Universidad de la capital de los países se asumía como universidad Mayor o Nacional. Siguiendo el modelo colonial de Universidades en los virreinatos o capitanías, promovió una estructuración universitaria jerárquica al interior de los países, tal como a su vez las universidades coloniales eran jerarquizadas en lo curricular a la Universidad de Salamanca en España. La creación de la Universidad Nacional de Colombia se produjo en el marco de un debate de su rol central frente a las otras universidades regionales. Los conceptos de universidad mayor, central, nacional o de la república en varios países, expresa este enfoque sistémico centralista en relación a las universidades descentralizadas o regionales sujetas a un ordenamiento jerárquico frente a la universidad de la capital del país.

Aún siendo autónomas las universidades regionales ellas son dependientes en el marco de un modelo centralizado. En este esquema de diferenciación de niveles, el acceso a la educación superior en las regiones de varios países, se ha soportado en institutos terciarios en los cuales la formación de capital humano se focaliza en niveles disciplinarios y epistemológicos inferiores. En las capitales es donde tienden a está instaladas las Universidades y en el interior los institutos terciarios. En la mayor parte de la región, y especialmente en Brasil, Argentina y Uruguay, este fraccionamiento de niveles de instituciones fue la base de los sistemas de educación superior. Este modelo de diferenciación mediante ámbitos de formación terciarios y universitarios como modelo binario que ha fragmentado la educación superior a escala nacional, y donde se ha localizado en el interior a la formación docente y la formación vocacional, técnica y tecnológica, en tanto que la profesional universitaria y la investigación de postgrado están localizadas en las capitales. La formación terciaria se ha constituido en el espacio dominante del acceso en el interior de muchos países. La universitarización de la formación docente y de la formación tecnológica es en este sentido un proceso de cambio en las formas de la regionalización de la educación superior.

La fragmentación en sub sedes y niveles también ha sido una diferenciación de la regulación, la calidad y el empoderamiento. Mientras que la regulación universitaria ha descansado en la autonomía y el cogobierno, o en organismos nacionales, la regulación terciaria a diferencia ha descansado en los Gobiernos, en general a través de los Ministerios de Educación (Panamá o Costa Rica) o en los gobiernos regionales (Argentina o México). 


\subsection{LOS MODELOS UNIVERSITARIOS DESCENTRALIZADOS}

Los modelos universitarios descentralizados territorialmente se basan en un paradigma que pone de relieve la valorización del acceso regional a través de universidades regionales como base de la construcción de las naciones y de mayores equilibrios territoriales. En general sus génesis se articularon a enfoques regionalizados de la construcción de las independencias como en la Gran Colombia que impulsó múltiples universidades a su interior (Trujillo, Popayán, Quito, Bogotá, Caracas, Cartagena, Nariño). Otros fueron proyectos claramente asociados a independencias como la Universidad Nacional de Loja en Ecuador. En otros casos inclusive han sido la base de proyectos de creación de estados independientes como se planteó en la ocasión de la creación de la Universidad de Antioquia en Medellín en Colombia (Burbano, 2008).

Las universidades regionales se soportaron en la búsqueda de construir capacidades regionales como base de un desarrollo nacional regional con instituciones en Departamentos, Estados, Provincias o Regiones y fueron expresión fines del Siglo XIX de económicos basados en la introducción de dinámicas capitalistas regionales apoyadas en exportaciones de productos primarios apoyados en ventajas competitivas que crearon importantes burguesías regionales que promovieron universidades como Cuenca (1867), Zulia (1891) o La Plata (1905). Inversamente el centralismo en México con el Porfiriato (Marsiske, 2006) y en Argentina limito estas expresiones regionales.

Algunas universidades regionales nacieron en el marco de modelos de evangelización en la época colonial mediante grupos religiosas que instalaron universidades asociadas a los conventos como Córdoba (Argentina), San Xavier (Bolivia), Huamanga (Perú) (Agueda, 2008). La expansión de las universidades asociada a los grupos religiosos y a su labor de evangelización continúo hasta el siglo XX pero ya en el ámbito privado y ha continuado impactando en la regionalización universitaria. Aunque nacieron como proyectos universitarios autónomos, en algunos países posteriormente se impuso al interior de sistemas universitarios una relativa jerarquización y dependencia al establecerse universidades Nacionales, Centrales o Mayores que ostentaban roles mayores.

Sin embargos estas universidades regionales descentralizadas, por sus bajos recursos y escalas, su encerramiento, o las limitaciones en un marco jerarquizado a la institucional nacional, no han alcanzado en general los mismos niveles de calidad que las universidades nacionales. En muchos casos al inicio carecieron de conexiones internacionales y sus niveles 
de formación se redujeron a pocas ofertas. En varios casos ello derivó en mayores impulsos para construir redes internacionales como mecanismo para salir del encerramiento de las regiones. Pero lo fundamental ha sido que las Universidades regionales han tenido altos niveles de empoderamiento y de articulación social y económica a sus ámbitos locales, tanto políticos, económicos como religiosos. Aunque en muchos casos dependieron de políticas nacionales que establecieron una jerarquización de los servicios educativos a escala nacional, como parte de los esquemas centralizados de los Estados latinoamericanos, las universidades regionales han impulsado mayores niveles de vinculación y empoderamiento local. Más recientemente además ellas han se han regionalizado internamente en subsedes en el tercer nivel del Estado. Así, las universidades localizadas en el segundo nivel de las estructuras del Estado en los Departamentos, Provincias, Departamentos o Estados, se han desconcentrado a su vez en el nivel municipal, en el tercer nivel del Estado. La ausencia de marcos globales o nacionales de aseguramiento de la calidad y sus autonomías relativas han facilitado mayores pertinencias locales. Sin embargo es necesario aclarar que también la desconcentración en el segundo nivel del Estado, no siempre han logrado escapar al círculo de la desigualdad educativa y la problemática de la calidad a escala local, con una dinámica centralizada en su relación con los niveles municipales jerarquizados del tercer nivel del Estado como ámbitos de su desconcentración. Uno de los componentes de esta regionalización, tanto como desconcentración o como descentralización, ha sido la búsqueda de conformar redes y cluster regionales internacionales para alcanzar niveles superiores. En tanto las regiones están más cerca entre ellas, se produce una regionalización y articulación transfronteriza. La alta porosidad de algunas partes de las fronteras, refuerza múltiples articulaciones regionales interfronterizas en la cual la movilidad estudiantil y docente adquiere dimensiones superiores tales que han permitido la movilidad académica y mayores escalas de matriculas.

\subsection{MODELOS UNIVERSITARIOS EN RED}

La construcción de capacidades locales en un contexto de diferenciación institucional, de escalas regionales reducidas y de creciente competencia por atracción de estudiantes, promueve un nuevo escenario de regionalización asociada a una concertación entre las distintas instituciones buscando la creación de sinergias y sistemas homogéneos de funcionamiento. Ello se expresa en una lógica de universidad en red sin vértice o centro 
dominante y donde las relaciones entre los nodos no son jerárquicas sino heterárquicas. (Figaredo, 2007).

La universidad en red ha sido concebida como un nuevo paradigma de la educación superior asociado a la educación virtual (Amador, 2006). Ello en tanto reproduce los tres principios sobre los cuales opera internet, dados por una estructura reticular, un poder de computación distribuido entre los diversos nodos y una redundancia de funciones en la red para minimizar riesgos de desconexión (Castells, 2001). Para este autor, en el análisis de Internet, para que se estructure una red deben cumplirse que la arquitectura debe ser de carácter abierto, descentralizado, distribuido y multidireccional en su interactividad, que los protocolos de comunicación deben ser abiertos, distribuirse libremente y ser susceptibles de modificación, y que las instituciones que gestionan la red deben construirse de acuerdo a los principios de transparencia y cooperación.

Estos criterios permiten concebir una universidad red. En su defecto, sino se cumplen, estaríamos frente a una estructura desconcentrada con mayor o menos nivel de dependencia o de independencia de los nodos de la red. Bajo una universidad red, su estructura reticular es más amplia y rica en interacciones en función de la cantidad de nodos o unidades de la institución dentro y fuera de un territorio particular. Con ello la innovación y la capacidad de creación de conocimiento y de efíciencia sistémica estarán dadas por la cantidad de interacciones entre los diversos nodos en igualdad de condiciones.

Para Amador, la universidad en red se plantea como un espacio de institucionalización y legitimación de la organización social de las comunidades que actúan a través de internet y donde el mayor protagonismo estará dado por las instituciones virtuales, no sólo por su tipo de acceso y su funcionamiento bajo redes tecnológicas, sino además por una dinámica de su gestión a través de redes telemáticas. Sostiene que "el paradigma informacional de las universidades en red presupone el desarrollo de las tecnologías de información y comunicación" (Amador, 2006, 168). La universidad red sin embargo, no se limita a las instituciones a distancia, sino a una dinámica de funcionamiento institucional, educativa y de gestión del conocimiento que enlaza los múltiples campus, facultades, regiones y centros de investigación, como base de la toma de decisiones y como eje de la gestión administrativa, académica y de investigación.

La universidad en red, reticular y distribuida en el poder, es la forma más eficiente de resolver la regionalización. El aumento de las unidades desconcentradas en la dinámica 
universitaria va deteriorando la calidad y la eficiencia. La necesidad de mantener e incrementar la eficiencia del sistema, en el marco de impulsos a la desconcentración institucional mediante sedes, va requiriendo e imponiendo un funcionamiento en red como mecanismo de integrar armónicamente el funcionamiento de los nodos y a la vez dotarse de eficiencias de cooperación y sinergías, con unidades independientes funcionando coordinadamente tanto con la sede central como entre ellas dentro de una estructura reticular. Los mecanismos de accesibilidad, los modelos educativos, los aprendizajes, la eficiencia y los sistemas de gestión se benefician de las estructuras de redes conformando dinámicas regionalizadas y de empoderamiento en la localización y en la pertinencia de los nodos.

En el plano regional la universidad red puede se puede conformar bajo lógicas de "cluster" para impulsar la creación de capacidades locales. El cluster es una de las formas que asume en el ámbito local, una dinámica educativa constituida alrededor de flujos y articulaciones compartidas donde bajo redes locales de trabajo académico, actores diversos y amplios niveles de concurrencia, pero que a la vez encuentran sinergias para ofertar servicios, compartir ventajas o potenciar iniciativas para reducir las debilidades del espacio local.

Esta estrategia se asocia también a la conformación de "ciudad universitaria" e inclusive al de "industria universitaria" (Pavez, 2006), para ofrecer bienes y servicios especializados orientados a sus habitantes universitarios, tales como hospedaje universitario, espacios para el esparcimiento, infraestructura recreativa y deportiva, grandes librerías, etc., y donde la ciudad sea capaz de generar polos de industria vinculados a la investigación universitaria y la "gestión del conocimiento" en el nivel local". Ello inclusive puede derivar en creación de estándares comunes de oferta, con lo cual los cluster sientan las bases para la conformación de subsistemas regionales de regulación de la educación superior.

El concepto de "cluster" como concentraciones de empresas e instituciones interconectadas en un campo particular para la competencia, ha sido propuesto por Porter (1991) para impulsar la competitividad de las empresas en el nuevo contexto de la globalización y de la necesidad de alcanzar umbrales mayores de competitividad, y se ha constituido en una referencia para los desarrollos regionales orientados a la exportación de servicios y el refuerzo a la creación de capacidades locales. Los enfoque de la investigación centrados en la famosa triple hélice (Estado, empresas, universidades) han pasado a incorporar una cuarta hélice y de su lógica territorial en comunidades locales como base contributiva para la creación de conocimiento. Ello remite a un grupo de instituciones 
interconectadas que logran alcanzar mayores ventajas a partir de una determinada localización y una articulación a los poderes locales y economías locales a partir de las externalidades que integradas ellas pueden generar y que les permite que se constituyan en motores dinámicos del desarrollo regional gracias al trabajo en red.

Esta es una de las formas nuevas de la regionalización de la educación superior en el actual contexto de la diferenciación de los sistemas de educación superior e incluye la participación de instituciones y dinámicas descentralizadas como desconcentradas al interior de las redes que las contienen y articulan, y cuyas capacidades, autonomías y beneficios están asociadas a sus tamaños y a la intensividad del uso de las tecnologías, de la comunicación horizontal y la articulación entre los componentes comunes de la red, los componentes independientes y de empoderamiento particulares, así como de apoyos locales, y también, aperturas globales.

\subsubsection{Las redes sociales desterritorializadas}

En el marco de dinámicas en red, ellas crecientemente asumen componentes virtuales como redes digitales y se constituyen en la nueva frontera de la reflexión educativa de relaciones entre tecnología y educación como espacio de circulación de los recursos de aprendizaje digitales a través de las redes sociales e institucionales (Moreira y Torres, 2015). La dinámica de redes facilitó una mayor descentralización y la conformación de lógicas de aprendizaje desterritorializadas en red.

Con las redes digitales, múltiples líneas de la investigación académica se han focalizado en su utilización pedagógica, buscando comprender mejor sus potencialidades como comunidades digitales de aprendizaje al interior de las redes sociales digitales. Esta línea de investigación toma del vasto mundo emergente de las tecnologías digitales, los aspectos más asociados a las redes sociales e institucionales, y se apoya en la sustentación teórica del conectivismo plateada por Siemens, Castells y Tapscott, centrándose en como las redes promueven comunidades como Facebook y otras como blog, wiki, listas de correo, etc., que se constituyen en estructuras de aprendizaje en tanto comunidades. Ellas son la base de una amplia dinámica de intercambio de información y de interacción de contenidos, con un rol central como industrial cultural en la actualización y socialización de la información. La masificación que ha adquirido Facebook y su carácter intraregional, nacional e internacional, otorga una especial atención a estas formas y a sus múltiples - y crecientes - impactos socio 
educativos, deconstruyendo el discurso tradicional de la regionalización y facilitando dinámicas y comunidades de aprendizaje regionales en red y creando verdaderos aprendizaje con sus propias características y sus diferenciados usos en el aprendizaje apoyando tanto dinámicas presenciales como virtuales.

\subsubsection{El modelo de desconcentración integrada}

La regionalización bajo formas desconcentradas o descentralizadas, o en el marco de una educación a distancia con apoyo local, tanto como unidades subsidiarias o autónomas, pero con relativas limitaciones de escalas, impulsa la oportunidad de construir dinámicas en red como mecanismo de alcanzar mejores niveles de eficiencia. La articulación de sinergias entre los diversos actores universitarios en un territorio local propende en una de sus orientaciones a la construcción de cluster entre las diversas instituciones. También hay agrupamientos gremiales, consorcios universitarios específicos o redes de universidades resultado de compras por grandes grupos globalizados. Con las formas en red tipo cluster aumentan las capacidades locales, se empoderan las instituciones, aumentando las externalidades de los limitados espacios locales. Analizaremos varios casos de cluster regionales que nos permiten visualizar estas expresiones en redes colaborativas de la regionalización. En la región encontramos entre otros el Cluster Punta del Este Ciudad Universitaria (Uruguay), el Cluster Valparaíso (Chile) y Manizales como ciudad Universitaria. Las limitaciones de las sedes desconcentradas o de las propias instituciones descentralizadas, se reducen mejorando sus capacidades y empoderamiento al trabajar en redes.

La nueva universidad no expresa meramente una regionalización descentralizada, sino también una evolución de la dinámica del empoderamiento, en tanto resultado de la transformación, en un ámbito regional específico de diversos institutos terciarios que pasaron a ser las patas creadoras de la Universidad. Por ejemplo, la universitarización de institutos terciarios regionales lo vemos en múltiples lados. El modelo de desconcentración integrado, como política pública de nivel nacional, también se ha expresado en la creación de los Centros Regionales de Educación Superior (CERES) de Colombia y en las Aldeas Universitarias de Venezuela. 


\section{NUEVAS FORMAS DE REGIONALIZACIÓN DE LA EDUCACIÓN SUPERIOR EN AMÉRICA LATINA:

\section{CONCLUSIONES}

La regionalización de la educación superior ha sido un proceso de tensiones entre dos esquemas de expresión marcados entre la descentralización y la desconcentración. Tales paradigmas han tenido sus fases y características diferenciadas, y se soportan en marcos conceptuales diferenciados. La evolución histórica de la universidad ha estado marcada por esas diferenciadas orientaciones. En el marco de los nuevos escenarios políticos, sociales, tecnológicos, educativos y conceptuales, irrumpe un nuevo paradigma basado en el concepto de red, que está promoviendo un nuevo modelo de regionalización universitario en red, que llamamos la universidad red, que supera la dualidad de sedes y universidades independientes para conformar una diversidad de modalidades de funcionamiento asociadas, en red, articuladas regionalmente, y buscando sinergías y eficiencias, y que al mismo tiempo promueve mayores niveles de empoderamiento y de dinámica articulada con los ámbitos públicos y comunitarios. Sin embargo, al tiempo se verifica la expansión de las universidades virtuales, o dinámicas articuladas de instituciones como parte de grupos nacionales e internacionales, que desarrollan lógicas de regionalización sin empoderamiento y con baja articulación territorial.

La Universidad red, como forma articulada entre instituciones de distinto nivel y sector, al tiempo impulsa la superación de las limitaciones de calidad, escala o apoyos que ha tenido la tradicional regionalización, muchas veces marcada por menores niveles de calidad. Estos formatos nuevos, unas de cuyas expresiones son los cluster, se producen al interior del sector público y privado, superando sus tradicionales fronteras y propenden a superar las debilidades de la educación superior en el interior de muchos países de la región por la vía de un mayor empoderamiento regional universitario.

\section{REFERENCIAS}

Amador, Rocío (2006). “La Universidad en red: un nuevo paradigma de la educación superior”. Revista Mexicana de Investigación Educativa. Vol. 11, № 28.

Barry, Luis Antonio (2005). La transferencia de los servicios educativos. Buenos Aires: Nuevohacer.

Burbano, Galo (2008), “Colombia” en C. García Guadilla (ed.) Pensadores y forjadores de la universidad latinoamericana. Caracas, CENDES, IESALC 
Castells (2012). "El poder en la era de las redes sociales". México, Nexos, setiembre. Disponible en http://www.nexos.com.mx/?p=14970. (Consultado el 20 de enero, 2015)

Castells, Manuel (2001). La galaxia internet. Reflexiones sobre Internet, empresa y sociedad. Madrid, Madrid

CEUB (Comité Ejecutivo de la Universidad Boliviana), (2014), Año III, No. 3, La Paz

Clark, Burton (2000) "Creando universidades innovadoras: estrategias organizacionales para la transformación”. México, UNAM, Porrúa

Estado de la Educación Costarricence (2011) programa Estado de la Nación en Desarrollo Humano Sostenible, San José, Consejo de Rectores, San José

Ezcurra, A.M. (2011). Igualdad en educación superior. Un desafío mundial. Buenos Aires. Universidad Nacional de General Sarmiento.

Figaredo, Daniel (2009). “La organización red en educación a distancia”. RIED, V. 12: 1, Freire, Pablo (1987). Pedagogía del Oprimido, México Siglo XXI Editores

Hanushek, Eric, (2011). "La importancia de la calidad en la educación”, en S. Cardus (2011) Los laberintos de la educación. España, Gedisa

Heredia, Blanca (2011). "Educación para el desarrollo", en S. Cardus (2011) Los laberintos de la educación. Gedisa, España

Huntington, Samuel y Crozer, Michel (1975). The crisis of democracy. Report on the Governability of Democracies to the Trilateral Commision. New York: University Press. Lundvall, (2002) "National systems of production, innovation and competence building". Research Policy 31. Disponible en http://infojustice.org/download/gcongress/dii/lundvall\%20article\%202.pdf (Consultado: 2 de febrero 2015)

Marsiske, Renate (2006). "La universidad de México: Historia y Desarrollo”. Revista Historia de la Educación Latinoamericana, núm. 8,

Martínez, Felipe (2002). La federalización de la educación superior en México. Alcances y limitaciones del proceso en la década de los años noventa. México, ANUIES

Menghini, Raúl Armando (2007). Universidades provinciales y nuevas formas de gestión. Buenos Aires, Universidad Nacional del Centro de la Provincia de Buenos Aires - Facultad de Ciencias Humanas.

Moncayo, Edgar (2002). Nuevos enfoques teóricos, evolución de las políticas regionales e impacto territorial de la globalización. Santiago de Chile, Instituto Latinoamericano y del Caribe de Planificación Económica y Social - ILPES. Serie de gestión pública No 27. 
Montecinos, Egon Elier (2013) Los actuales desafíos regionales en Chile: ¿Nueva Regionalización o más Descentralización?. Los Lagos Universidad de Los Lagos, Centro de Estudios del Desarrollo Regional y Local (CEDER). Disponible en http://achm.cl/eventos/seminarios/html/documentos/2013/XI ASAMBLEA DE MUNICIPA LIDADES/CONGRESO/PPT10.pdf (Consultado, 25 de enero, 2015)

Moreira Matos, Elizete y Lupion Torres, Patricia (2015). Redes E midias sociais Curitiba. PUCP

Pavez, Natalia (2007) "El concepto de ciudad universitaria". Disponible en http://nataliapavez.blogspot.com/2007/10/concepto-de-ciudad-universitaria.html (Consultado 30 de octubre de 2014)

Porter, Michael (1991). La ventaja competitiva de las naciones. Barcelona, Plaza \& Janes

Putnam, R. (1993) "The prosperous community. Social capital and public life". The American theory and the social capital debate". American Prospect, vol.4, ${ }^{\circ} 13$

Rama, Claudio. (2009). La tendencia a la masificación de la cobertura de la educación superior en América Latina. Revista Iberoamericana de Educación, 50 (Julio), pp. 173-195.

Rangel, Rafael (2014). Universidad 2.0. México, Taurus

Rodríguez Cruz, Águeda (2008), "Protagonismo de la universidad de Salamanca en los pensadores y forjadores de las universidades hispanoamericanas", en C. García Guadilla (ed.) Pensadores y forjadores de la universidad latinoamericana. Caracas, CENDES, IESALC

Solana, Fernando. (compilador). Educación y desigualdad. Siglo XXI, México, 2005

Urteaga, Eguzki (1993) "La teoría del capital social de Robert Putnam: Originalidad y carencias” Reflexión Política, vol. 15, núm. 29. 